\title{
Some Embeddings into the Multiplier Spaces Associated to Besov and Lizorkin-Triebel Spaces
}

\author{
D. Drihem and M. Moussai
}

\begin{abstract}
We study the set of pointwise multipliers in the Lizorkin-Triebel space $F_{p}^{s, q}$ and of the corresponding multiplier set in the Besov space $B_{p}^{s, q}$, where we give sufficient conditions on the parameters $s, p$ and $p_{1}$ such that the embeddings $F_{p_{1}}^{n / p_{1}, \infty} \cap L^{\infty} \hookrightarrow M\left(F_{p}^{s, q}\right)$ and $B_{p_{1}}^{n / p_{1}, \infty} \hookrightarrow M\left(B_{p}^{s, q}\right)$ hold.
\end{abstract}

Keywords: Besov spaces, Lizorkin-Triebel spaces, pointwise multipliers

AMS subject classification: 46E 35

\section{Introduction}

We propose a study of set $M\left(F_{p}^{s, q}\right)$ of pointwise multipliers in the LizorkinTriebel space $F_{p}^{s, q}$ and of the corresponding multiplier set in the Besov space $B_{p}^{s, q}$. Let us recall that

- $M\left(F_{p}^{s, 2}\right)=F_{p, \text { unif }}^{s, 2}\left(1<p<\infty, s>\frac{n}{p}\right)$ (Strichartz [9]).

- $M\left(B_{p}^{s, p}\right)=B_{p, \text { unif }}^{s, p} \quad\left(1 \leq p \leq \infty, s>\frac{n}{p}\right)$ (Peetre [6]).

- $M\left(B_{p}^{s, p}\right) \quad(1 \leq p \leq \infty, s>0)$ has been characterized in terms of capacities by Maz'ya and Shaposnikova [5].

- $M\left(F_{p}^{s, q}\right)=F_{p, \text { unif }}^{s, q} \quad\left(1 \leq p<\infty, 1 \leq q \leq \infty, s>\frac{n}{p}\right)$ (Franke [2]).

- $M\left(B_{p}^{s, q}\right) \neq B_{p, \text { unif }}^{s, q} \quad\left(1 \leq q<p \leq \infty, s>\frac{n}{p}\right)$ (Bourdaud [1]).

- $M\left(B_{1}^{s, q}\right) \quad(1 \leq q \leq \infty, s>0)$ has been characterized in Fourier analytic terms by Netrusov (see, for example, [7]).

- $M\left(B_{p}^{s, q}\right)=B_{p, u n i f}^{s, q} \quad\left(1 \leq p \leq q \leq \infty, s>\frac{n}{p}\right)$ (Sickel and Smirnov [9]).

In this paper we consider essentially the case $s=\frac{n}{p}$ and this contribution is the continuation of Runst and Sickel's work [7].

Both authors: M'Sila Univ., Dept. Math., P.O. Box 166, M'Sila 28000, Algeria mmoussai@yahoo.fr

ISSN 0232-2064 / \$2.50 C Heldermann Verlag Berlin 


\section{Preliminaries}

All functions, spaces etc. are defined on the Euclidean space $\mathbb{R}^{n}$. We set $\mathcal{D}=\mathcal{D}\left(\mathbb{R}^{n}\right), L^{p}=L^{p}\left(\mathbb{R}^{n}\right)$ etc. If $f \in \mathcal{S}$, then

$$
\mathcal{F} f(\xi)=\hat{f}(\xi)=\frac{1}{(2 \pi)^{n}} \int_{\mathbb{R}^{n}} f(x) \exp (-i x \cdot \xi) d x \quad\left(\xi \in \mathbb{R}^{n}\right)
$$

denotes the Fourier transform of $f$ and $\mathcal{F}^{-1} f$ its inverse transform.

Let $\phi \in \mathcal{D}$ such that $\phi \geq 0, \operatorname{supp} \phi \subset\left\{\xi \in \mathbb{R}^{n}: 1 \leq|\xi| \leq 3\right\}$ and $\sum_{j \in \mathbb{Z}} \phi\left(2^{-j} \xi\right)=1$. It follows that the function $\xi \rightarrow \varphi(\xi)=1-\sum_{j \geq 1} \phi\left(2^{-j} \xi\right)$ is in $C^{\infty}$ with support in the ball $|\xi| \leq 3$ and one has $\varphi(\xi)+\sum_{j \geq 1} \phi\left(2^{-j} \xi\right)=$ $1\left(\xi \in \mathbb{R}^{n}\right)$. To this partition of unity we associate the convolution operators $\Delta_{k}(k \in \mathbb{N})$ and $Q_{j} \quad(j \in \mathbb{N} \cup\{0\})$ defined by

$$
\left(\Delta_{k} f\right)^{\wedge}(\xi)=\phi\left(2^{-k} \xi\right) \hat{f}(\xi) \quad \text { and } \quad\left(Q_{j} f\right)^{\wedge}(\xi)=\varphi\left(2^{-j} \xi\right) \hat{f}(\xi) .
$$

We set $\Delta_{0}=Q_{0}$. The Littlewood-Paley decomposition is the identity

$$
f=Q_{k} f+\sum_{j \geq k+1} \Delta_{j} f \quad\left(Q_{k} f=\sum_{j \leq k} \Delta_{j} f\right)
$$

of all $f \in \mathcal{S}^{\prime}$. The series converges in $\mathcal{S}^{\prime}$.

The support of $\Delta_{k}\left(\Delta_{j} f \Delta_{l} g\right)$ is not empty in one of the following cases:

$$
\begin{array}{ll}
l \leq k+1 & \text { and } k-2 \leq j \leq k+4 \\
j \leq k+1 & \text { and } k-2 \leq l \leq k+4 \\
l, j \geq k & \text { and }|l-1| \leq 1
\end{array}
$$

Then we can write the product

$$
f g=\sum_{k \geq 0}\left(\Delta_{k(1)}+\Delta_{k(2)}+\Delta_{k(3)}\right)(f g)
$$

where

$$
\begin{aligned}
& \Delta_{k(1)}(f g)=\Delta_{k}\left(\widetilde{\Delta}_{k} f \cdot Q_{k+1} g\right) \\
& \Delta_{k(2)}(f g)=\Delta_{k}\left(Q_{k+1} f \cdot \widetilde{\Delta}_{k} g\right) \\
& \Delta_{k(3)}(f g)=\sum_{j \geq k} \Delta_{k}\left(\Delta_{j} f \cdot \bar{\Delta}_{j} g\right)
\end{aligned}
$$

with $\widetilde{\Delta}_{k}=\sum_{j=k-2}^{k+4} \Delta_{j}$ and $\bar{\Delta}_{k}=\sum_{j=k-1}^{k+1} \Delta_{j}$.

Now, we recall the definition of Besov and Lizorkin-Triebel spaces. For more details about equivalent norms, embeddings etc. see $[6,7,10]$. 
Definition 2.1. For $s \in \mathbb{R}$ and $1 \leq p, q \leq \infty$ the Besov space is

$$
B_{p}^{s, q}=\left\{f \in \mathcal{S}^{\prime}:\left(\sum_{j \geq 0} 2^{s j q}\left\|\Delta_{j} f\right\|_{p}^{q}\right)^{\frac{1}{q}}<\infty\right\}
$$

For $s \in \mathbb{R}, 1 \leq p<\infty$ and $1 \leq q \leq \infty$ the Lizorkin-Triebel space is

$$
F_{p}^{s, q}=\left\{f \in \mathcal{S}^{\prime}:\left\|\left(\sum_{j \geq 0} 2^{s j q}\left|\Delta_{j} f\right|^{q}\right)^{\frac{1}{q}}\right\|_{p}<\infty\right\} .
$$

We will use the following assertions throughout the paper.

Lemma 2.1. If $0<\delta<1$ and $1 \leq q \leq \infty$, then for every sequence $\left(\varepsilon_{j}\right)_{j \in \mathbb{N}} \in \ell^{q}$ of positive numbers one has

$$
\left\|\left(\delta^{j} \sum_{k \leq j} \delta^{-k} \varepsilon_{k}\right)_{j}\right\|_{\ell^{q}}+\left\|\left(\delta^{-j} \sum_{k \geq j} \delta^{k} \varepsilon_{k}\right)_{j}\right\|_{\ell^{q}} \leq \frac{2}{1-\delta}\left\|\left(\varepsilon_{j}\right)_{j}\right\|_{\ell^{q}} .
$$

Lemma 2.2 (Bernstein's inequality). If $1 \leq p \leq q \leq \infty$ and $\alpha \in \mathbb{N}^{n}$, then there exists a constant $C>0$ such that

$$
\left\|f^{(\alpha)}\right\|_{q} \leq C R^{|\alpha|+n\left(\frac{1}{p}-\frac{1}{q}\right)}\|f\|_{p}
$$

for all $f \in L^{p}$ with $\operatorname{supp} \hat{f} \subset\left\{\xi \in \mathbb{R}^{n}:|\xi| \leq R\right\}$.

Inequality (2) follows by using Young's inequality in $\ell^{q}$. Similarly, for (3) we apply Young's inequality to $f^{(\alpha)}=\theta_{R}^{\alpha} * f$ where $\theta_{R}(x)=R^{n} \theta(R x) \quad(x \in$ $\left.\mathbb{R}^{n}, R>0\right)$ such that $\theta \in C^{\infty}$ and $\hat{\theta}(\xi)=1$ if $|\xi| \leq 1$.

We finish now this section by recalling the definition of the pointwise multipliers space of a Banach space $E$ (in our work $E=F_{p}^{s, q}$ or $E=B_{p}^{s, q}$ ), denoted by $M(E)$. This is the set of all functions $m$ such that $\|m f\|_{E} \leq$ $C\|f\|_{E}(f \in E) . M(E)$ is a Banach space with the norm equal to the infinum of the above constants $C$. Concerning the properties of $M\left(F_{p}^{s, q}\right)$ and $M\left(B_{p}^{s, q}\right)$ we do not go into details, referring the reader to $[2,6,7]$. 


\section{Embedding into $M\left(F_{p}^{s, q}\right)$}

In this section we shall formulate the result for Lizorkin-Triebel space.

Theorem 3.1. Let $s \in \mathbb{R}, 1 \leq p \leq p_{1}<\infty, 1 \leq q \leq \infty, r \geq \frac{n}{p_{1}}$ and $\frac{n}{p_{1}}-r+\frac{n}{p}-n<s<r$. Then

$$
F_{p_{1}}^{r, \infty} \cap L^{\infty} \hookrightarrow M\left(F_{p}^{s, q}\right) .
$$

Proof. We treat only the case $r=\frac{n}{p_{1}}$. The case $r>\frac{n}{p_{1}}$ is given in [7: Subsections 4.4.3 and 4.4.4] and the papers of Marschall [3,4]. Let $f \in F_{p}^{s, q}$ and $g \in F_{p_{1}}^{\frac{n}{p_{1}}, \infty} \cap L^{\infty}$. For the estimate $\|f g\|_{F_{p}^{s, q}}$ we need decomposition (1) and the maximal inequality

$$
\left\|\left(\sum_{k \geq 0}\left(\Delta_{k}^{*, a} f\right)^{q}\right)^{\frac{1}{q}}\right\|_{p} \leq C\left\|\left(\sum_{k \geq 0}\left|\Delta_{k} f\right|^{q}\right)^{\frac{1}{q}}\right\|_{p}
$$

satisfied for all $f \in \mathcal{S}^{\prime}$ and $a>\frac{n}{\min (p, q)}$, where $\left(\Delta_{k}^{*, a} f\right)(x)=\sup _{y \in \mathbb{R}^{n}} \frac{\left|\left(\Delta_{k} f\right)(x-y)\right|}{\left(1+\left|2^{k} y\right|\right)^{a}}$ (see [10: Theorem 2.3.6] or [7]).

Estimate of $\Delta_{k(1)}(f g)$. Let us set

$$
C=\int_{\mathbb{R}^{n}}\left|\left(\mathcal{F}^{-1} \phi\right)(y)\right|(1+|y|)^{a} d y .
$$

Since

$$
\left\|Q_{k+1} g\right\|_{\infty} \leq C\|g\|_{\infty}
$$

we obtain $\left|\Delta_{k(1)}(f g)\right| \leq C\|g\|_{\infty}\left(\Delta_{k}^{*, a} f\right)$. Taking $a>\frac{n}{\min (p, q)}$ and applying (4) yield

$$
\left\|\left(\sum_{k \geq 0} 2^{s q k}\left|\Delta_{k(1)}(f g)\right|^{q}\right)^{\frac{1}{q}}\right\|_{p} \leq C\|g\|_{\infty}\|f\|_{F_{p}^{s, q} .}
$$

Estimate of $\Delta_{k(2)}(f g)$. We consider the case $p<p_{1}$. Let $a_{1}$ and $a_{2}$ in $\mathbb{R}^{+}$such that

$$
\left|\Delta_{k(2)}(f g)\right| \leq C\left(\Delta_{k}^{*, a_{1}} g\right) \sum_{j \leq k+1} \Delta_{j}^{*, a_{2}} f .
$$

By Lemma 2.1 we have

$$
\left(\sum_{k \geq 0} 2^{s q k}\left|\Delta_{k(2)}(f g)\right|^{q}\right)^{\frac{1}{q}} \leq C\left(\sum_{k \geq 0} 2^{k q\left(s-\frac{n}{p_{1}}\right)}\left(\Delta_{k}^{*, a_{2}} f\right)^{q}\right)^{\frac{1}{q}} \sup _{l \geq 0}\left(2^{l \frac{n}{p_{1}}} \Delta_{l}^{*, a_{1}} g\right) .
$$


Combining Hölder's inequality (where $\frac{1}{p}=\frac{1}{p_{1}}+\frac{1}{p_{2}}$ ) with (4) by taking $a_{1}>\frac{n}{p_{1}}$ and $a_{2}>\frac{n}{\min \left(p_{2}, q\right)}$ shows that the left-hand side of (6) in $L^{p}$-norm is bounded by

$$
C\|g\|_{F_{p_{1}}}^{\frac{n}{p_{1}}, \infty}\|f\|_{F_{p_{2}}^{s-\frac{n}{p_{1}}}, q}
$$

and it remains to use the inclusion $F_{p}^{s, q} \hookrightarrow F_{p_{2}}^{s-\frac{n}{p_{1}}, q}$.

Now we study the case $1 \leq p=p_{1}<\infty$. Let $v>1$ such that $1<p<v<$ $\infty$ and $s-\frac{n}{p}+\frac{n}{v}=s_{1}<0$. Set $\frac{1}{u}=\frac{1}{p}+\frac{1}{v}$. Then by Hölder's inequality

$$
\begin{aligned}
2^{\left(s+\frac{n}{v}\right) k}\left\|\Delta_{k(2)}(f g)\right\|_{u} & \leq C 2^{\left(s+\frac{n}{v}\right) k}\left\|\widetilde{\Delta}_{k} g\right\|_{p} \sum_{j \leq k+1}\left\|\Delta_{j} f\right\|_{v} \\
& \leq C\|g\|_{B_{p}^{\frac{n}{p}, \infty}} 2^{s_{1} k} \sum_{j \leq k+1} 2^{-s_{1} j}\left(2^{s_{1} j}\left\|\Delta_{j} f\right\|_{v}\right) .
\end{aligned}
$$

By applying Lemma 2.1 we obtain

$$
\left(\sum_{k \geq 0} 2^{\left(s+\frac{n}{v}\right) k p}\left\|\Delta_{k(2)}(f g)\right\|_{u}^{p}\right)^{\frac{1}{p}} \leq C\|g\|_{B_{p}^{\frac{n}{p}, \infty}}\|f\|_{B_{v}^{s_{1}, p} .}
$$

Since $B_{u}^{s+\frac{n}{v}, p} \hookrightarrow F_{p}^{s, q} \hookrightarrow B_{v}^{s_{1}, p}$ and $F_{p}^{\frac{n}{p}, \infty} \hookrightarrow B_{p}^{\frac{n}{p}, \infty}$ we obtain the desired estimation.

Estimate of $\Delta_{k(3)}(f g)$. The difficult part of the product is given by $\sum_{k \geq 0} \Delta_{k(3)} f g$. To get a bound for the norm of this expression one may use [7: Proposition 4.4.2/4(i)]:

$$
\left\|\sum_{k \geq 0} \Delta_{k(3)}(f g)\right\|_{F_{t}^{s+\frac{n}{p_{1}}, \infty}} \leq C\|g\|_{F_{p_{1}} \frac{n}{p_{1}}, \infty}\|f\|_{F_{p}^{s, q}}
$$

where $\frac{1}{t}=\frac{1}{p}+\frac{1}{p_{1}}$ and $s+\frac{n}{p_{1}}>n \max \left(0, \frac{1}{t}-1\right)$ is needed. This gives the correct bound for $s$ (see the necessary conditions in [7: Section 4.3]) in view of the embedding $F_{1}^{n, \infty} \hookrightarrow F_{p_{1}}^{\frac{n}{p_{1}}, \infty}$. Observe that $F_{t}^{s+\frac{n}{p_{1}}, \infty} \hookrightarrow F_{p}^{s, q}$

Remark 3.1. It is well known that the Hölder-Zygmund space $\mathcal{C}^{\rho}$ is not included in $M\left(F_{p}^{s, q}\right)$ for $0<\rho<|s|$ (see [10: p. 143]). Hence, if $1 \leq p \leq p_{1}<$ $\infty, r \geq \frac{n}{p_{1}}$ and $\frac{n}{p_{1}}-r+\frac{n}{p}-n<s<r$, then $\mathcal{C}^{\rho} \backslash F_{p_{1}}^{r, \infty} \nsubseteq M\left(F_{p}^{s, q}\right)$. 


\section{Embedding into $M\left(B_{p}^{s, q}\right)$}

We give now the corresponding result for $B_{p}^{s, q}$, where the following theorem improves the previous results obtained in [6: p. 146], [7: p. 173] and [10: p. $154]$.

Theorem 4.1. Let $s \in \mathbb{R}, 1 \leq p \leq p_{1} \leq \infty, 1 \leq q \leq \infty, r \geq \frac{n}{p_{1}}$ and $\frac{n}{p_{1}}-r+\frac{n}{p}-n<s<r$. Then

$$
B_{p_{1}}^{r, \infty} \cap L^{\infty} \hookrightarrow M\left(B_{p}^{s, q}\right) .
$$

Proof. As in the proof of Theorem 3.1, we consider only the case $r=\frac{n}{p_{1}}$. Let $f \in B_{p}^{s, q}$ and $g \in B_{p_{1}}^{\frac{n}{p_{1}}, \infty} \cap L^{\infty}$. We will estimate $\|f g\|_{B_{p}^{s, q}}$ by using (1). We systematically use the fact that $\Delta_{k}$ and $Q_{k}$ are bounded operators in $\mathcal{L}\left(L^{p}, L^{p}\right)$.

Estimate of $\Delta_{k(1)}(f g)$. We begin by

$$
\left\|\Delta_{k(1)}(f g)\right\|_{p} \leq C\left\|\widetilde{\Delta}_{k} f\right\|_{p}\left\|Q_{k+1} g\right\|_{\infty} .
$$

Then (5) and (8) give the desired estimation.

Estimate of $\Delta_{k(2)}(f g)$. The fact that $\left\|\Delta_{j} f\right\|_{p_{2}} \leq C 2^{j n\left(\frac{1}{p}-\frac{1}{p_{2}}\right)}\left\|\Delta_{j} f\right\|_{p}$ (Lemma (2.2) where $\frac{1}{p}=\frac{1}{p_{1}}+\frac{1}{p_{2}}$ and Hölder's inequality imply

$$
\begin{aligned}
2^{s k}\left\|\Delta_{k(2)}(f g)\right\|_{p} & \leq C\|g\|_{B_{p_{1}}^{\frac{n}{p_{1}}, \infty}} 2^{k\left(s-\frac{n}{p_{1}}\right)} \sum_{j \leq k+1} 2^{j \frac{n}{p_{1}}}\left\|\Delta_{j} f\right\|_{p} \\
& \leq C\|g\|_{B_{p_{1}}^{\frac{n}{p_{1}}, \infty}} 2^{k\left(s-\frac{n}{p_{1}}\right)} \sum_{j \leq k+1} 2^{j\left(\frac{n}{p_{1}}-s\right)}\left(2^{j s}\left\|\Delta_{j} f\right\|_{p}\right) .
\end{aligned}
$$

We conclude by Lemma 2.1 (since $s<\frac{n}{p_{1}}$ ).

Estimate of $\Delta_{k(3)}(f g)$. As in (7) we have

$$
\left\|\sum_{k \geq 0} \Delta_{k(3)}(f g)\right\|_{B_{t}^{s+\frac{n}{p_{1}}, \infty}} \leq C\|g\|_{B_{p_{1}}^{\frac{n}{p_{1}}, \infty}}\|f\|_{B_{p}^{s, q}}
$$

where $\frac{1}{t}=\frac{1}{p}+\frac{1}{p_{1}}$ and $s+\frac{n}{p_{1}}>n \max \left(0, \frac{1}{t}-1\right)$ is needed. In [7] only the limit case $s+\frac{n}{p_{1}}=n \max \left(0, \frac{1}{t}-1\right)$ is considered, but (9) is in the same spirit

Remark 4.1. As in Remark 3.1, if $0<\rho<|s|, 1 \leq p \leq p_{1} \leq \infty, r \geq \frac{n}{p_{1}}$ and $\frac{n}{p_{1}}-r+\frac{n}{p}-n<s<r$, then $\mathcal{C}^{\rho} \backslash B_{p_{1}}^{r, \infty} \nsubseteq M\left(B_{p}^{s, q}\right)$.

Acknowledgement. The authors are deeply grateful to Professor G. Bourdaud (University of Paris 6) for the suggestions and corrections. Also, the authors express their gratitude to the referee for the most helpful remarks which led to improvements of the results and presentation of the paper. 


\section{References}

[1] Bourdaud, G.: Localisations des espaces de Besov. Studia Math. 90 (1988), $153-163$.

[2] Franke, J.: On the spaces $F_{p}^{s, q}$ of Triebel-Lizorkin type: Pointwise multipliers and spaces on domains. Math. Nachr. 125 (1986), $29-68$.

[3] Marschall, J.: On the boundedness and compactness of nonregular pseudodifferential operators. Math. Nachr. 175 (1995), $231-262$.

[4] Marschall, J.: Remarks on nonregular pseudodifferential operators. Z. Anal. Anw. 15 (1996), 109 - 148.

[5] Maz'ya, V. G. and T. O. Shaposnikova: Theory of Multipliers in Spaces of Differentiable Functions. Boston: Pitman 1985.

[6] Peetre, J.: New Thoughts on Besov Spaces. Durham: Duke Univ. Math. Series 1976.

[7] Runst, T. and W. Sickel: Sobolev Spaces of Fractional Order, Nemytskii Operators and Nonlinear Partial Differential Equations. Berlin: de Gruyter 1996.

[8] Sickel, W. and I. Smirnov: Localization properties of Besov spaces and of its associated multiplier spaces. Jena (Germany): Jenaer Schriften Math/Inf/99/21.

[9] Strichartz, R. S.: Multipliers on fractional Sobolev spaces. J. Math. Mech. 16 (1967), 1031 - 1060.

[10] Triebel, H.: Theory of Function Spaces. Basel: Birkhäuser Verlag 1983.

Received 05.06.2001; in revised form 07.09.2001 